\title{
INDICADORES Y ESTÁNDARES INTERNACIONALES DE CALIDAD UNIVERSITARIA
}

\section{Introducción}

El estudio que da origen a este documento ha sido elaborado con el fin de otorgar material de referencia sobre las principales metodologías usadas internacionalmente para medir la calidad de una universidad. Para ello, se han analizado dos tipos de evaluaciones: i) rankings universitarios (publicados principalmente por revistas de circulación masiva) y ii) procesos de acreditación universitaria. En esta oportunidad se expondrán los antecedentes relativos al primer tipo de evaluación.

En general, los rankings están dirigidos a postulantes universitarios y sus familias. Los procesos acreditadores, en tanto, están más vinculados con el esfuerzo estatal de velar por la calidad de la educación superior. Ambos métodos difieren principalmente en que los primeros privilegian una evaluación cuantitativa, mientras que los procesos acreditadores se orientan más por una metodología cualitativa.

La metodología de investigación para elaborar este documento ha consistido principalmente en revisión bibliográfica y consultas directas a instituciones y publicaciones.

El eje de este estudio ha sido la experiencia internacional, por lo tanto, no se han analizado ni los rankings universitarios nacionales ni el proceso de acreditación chileno. 
18 INDICADORES Y ESTÁNDARES INTERNACIONALES DE CALIDAD UNIVERSITARIA - Rodrigo Fernández, Yerko Martínez, Nicolás Velasco

\section{Indicadores de calidad}

En el intento por medir y comparar la calidad de una universidad surgen varias discusiones: la relevancia y el objetivo de hacerlo (considerando que la educación universitaria es un bien no homogéneo), la calidad con que se lleva a cabo dicha medición (la propiedad y relevancia de los indicadores utilizados), el sesgo (cada ranking o indicador está diseñado con un fin específico -ya sea para estudiantes, organismos de gobierno, por ejemplo- y, por lo tanto, persiguen fines distintos).

La necesidad de medir la calidad surge de varias razones. En el caso de los rankings universitarios, el interés es, principalmente, proveer información a estudiantes sobre las universidades a las que desean postular. Además, ofrecen un mecanismo simple y de fácil entendimiento a través del cual la sociedad civil evalúa y controla la calidad de la educación superior.

Por tanto, rankings y agencias existen por razones distintas: los primeros son elaborados para los medios masivos y, por tanto, requieren ser fácilmente comprensibles y accesibles. No intentan ser exhaustivos en sus indagaciones sino más bien sintéticos. Las agencias de acreditación intentan dar cuenta de una evaluación más exhaustiva de la calidad de una institución de educación superior, y están destinadas a colaborar con las determinaciones de los policymakers de la sociedad respecto de estas materias.

Los rankings universitarios, a pesar de los cuestionamientos teóricos y metodológicos que se les pueda hacer, son importantes agentes en la creación de prestigio, una variable que no sólo es útil para atraer buenos estudiantes, sino también buenos profesores y fondos (donaciones y otros). 


\section{Indicadores utilizados por rankings universitarios internacionales en publicaciones masivas}

\section{Consideraciones preliminares}

Los rankings universitarios tienen por objeto realizar una jerarquización de universidades basándose en parámetros que intentan medir la calidad de la educación, el nivel de investigación y otros aspectos de la actividad académica, en un esfuerzo por informar y orientar a la opinión pública.

Sin embargo, cada universidad se constituye y organiza de acuerdo a sus propias prioridades y metas. No generan "productos" homogéneos y, en su mayoría, obedecen a misiones que les son propias y, por tanto, distintas entre ellas. De esta forma, al medirlas según parámetros comunes no se acoge completamente esta realidad y podría inducirse a errores de interpretación.

Por eso, es importante destacar que los indicadores construidos con estos fines tienen, en su concepción, un objetivo particular y distinto al de orientar el plan de desarrollo particular de una universidad. Para el cumplimiento de esa finalidad es más recomendable la construcción de indicadores propios.

Los rankings universitarios han sido elaborados, en su mayoría, como una guía para los postulantes en la elección de la institución en la cual proseguirán sus estudios, por lo tanto, su orientación está dirigida hacia la calidad de la educación que se ofrece. Sin embargo, detrás de variables como "gasto agregado", "gasto en investigación", "razón profesores/estudiantes", entre otras, subyace el supuesto (discutido en alguna literatura de la economía de la educación) de que mayores niveles de gasto implican mayor calidad de la educación impartida. Y no es preocupación de estos rankings medir cuán eficiente es el manejo de los recursos que hace una universidad en particular.

Una incipiente discusión acerca de su efecto sobre el desempeño de las universidades ha comenzado en EE.UU. Existe controversia sobre 
el impacto que tienen dichas jerarquizaciones (que se vuelven más populares año tras año) sobre las instituciones de educación superior. Las más prestigiosas de éstas no observan mayor cambio en la cantidad de postulaciones que reciben. Sin embargo, varias otras universidades aseguran que la comparación sí importa. Algunos estudios señalan que los rankings sí tienen impacto, pero acotado. En una encuesta realizada por The Higher Education Research Institute, sólo el 8,6\% de los estudiantes de primer año señaló que los publicados en revistas fueron determinantes al momento de elegir una universidad ${ }^{1}$.

La elaboración de todo ranking universitario es cuestionable desde distintos puntos de vista:

- Carecen de un sustento teórico fuerte respecto de su concepción de lo que significa "calidad" y, por lo mismo, sus metodologías -incluso las más prestigiosas, como la de U.S. News \& World Report- son acusadas de subjetivas y arbitrarias.

- La calidad de los datos o si éstos indican efectivamente lo que se quiere representar, son cuestionamientos que todo ranking debe enfrentar.

- La ponderación de cada variable es, en general, una elección arbitraria de quienes elaboran el ranking según los aspectos que a ellos les parece más importante destacar. Esto hace que la posición relativa de una universidad en particular sea altamente sensible a la combinación de ponderaciones elegida.

- La elección misma de las variables varía de un ranking a otro sin mayor justificación. Su elección parece arbitraria y, por tanto, el resultado es muy sensible a dicha elección.

- Por la misma naturaleza de sus objetivos, muchos componentes que conforman la "calidad" de una universidad no son cuantificables ${ }^{2}$.

1 Crissey, Michael (1997) Changes in Annual College Guide Fail to Quell Criticisms on Their Validity. The Chronicle of Higher Education, September 5. Available at http://chronicle. $\mathrm{com} /$ free/v44/i02/02a06701.htm

2 Kersten, Glenn (2000) Grading on the Curve: Collage Rating and Rankings. January. Available at http://www.sls.lib.il.us/reference/por/features/99/collrank.htm. 
En términos más específicos, la comunidad académica internacional no ha cesado de plantear múltiples y variadas críticas a su elaboración. Entre otras cosas se sugiere que:

- Jerarquizar universidades es un esfuerzo engañoso e impreciso. El lugar de las universidades en los rankings cambia de año en año con demasiada frecuencia. No es creíble que la calidad de una universidad cambie con tanta rapidez.

- El uso de algunas variables es inadecuado e impreciso. Por ejemplo, indicadores como razón de número de graduados esperados vs. número de graduados reales pueden estar castigando a las universidades que son más exigentes en la entrega del título.

- En un sistema que quiere estimular la diversidad de sus instituciones, una lista que mide estandarizadamente lo que considera "las mejores universidades" no resulta de mucha ayuda. Además, lo que es importante para un postulante puede no serlo para otro.

- Las diferencias en la posición ocupada pueden ser estadísticamente insignificantes, es decir, puede haber muy poca diferencia en "calidad" entre una entidad ubicada en el lugar 2 y otra en el 20, pero un lector desinformado piensa de otra manera.

Sin embargo, siguen siendo muy utilizados y cada vez más extensamente. Las mismas instituciones académicas han pasado de "un estado de abierta hostilidad a uno de disgusto disimulado"3.

Se puede decir que, pese a las numerosas críticas, se siguen utilizando debido a que:

- Las publicaciones ofrecen un servicio que es percibido como importante por el público en general.

- Por lo mismo, la mayor parte de la información que utilizan es provista por las mismas instituciones.

- Han resultado un negocio provechoso para las revistas.

3 Sharp, William Building reputations: How the game is played. 21stC. Research at Columbia. Available at http://www.columbia.edu/cu/21stC/issue-1.1/vying.htm 
- Muchos tienen méritos en la medida en que los estudiantes sean cautelosos en su interpretación.

- Los postulantes buscan una forma rápida y fácil para comparar las miles de escuelas disponibles. A pesar de que, sobre la decisión final, sigan pesando más las fuentes de información "humanas" (padres, amigos y compañeros de colegio, visitas estudiantiles, entre otras), los rankings ofrecen una manera de restringir las opciones a un número prudente y manejable.

\section{Análisis de rankings}

Se analizan 12 jerarquizaciones de EE.UU., Australia, Reino Unido y Asia, se les clasifica según tres directrices y, finalmente, se extraen las variables más comunes y representativas.

\subsection{Definición de directrices de análisis}

Directrices de análisis:

\begin{tabular}{l|l}
\hline COBERTURA & \\
\hline \multirow{3}{*}{ Geográfica } & $\begin{array}{l}\text { Mundial } \\
\text { Regional } \\
\text { Nacional }\end{array}$ \\
\hline \multirow{3}{*}{ Disciplinar } & $\begin{array}{l}\text { Global } \\
\text { Disciplinar } \\
\text { Unidisciplinar }\end{array}$ \\
\hline \multirow{2}{*}{ De ámbito } & $\begin{array}{l}\text { Pregrado (o si se incluye magíster, profesional) } \\
\text { Posgrado } \\
\text { Investigación (producción científica e innovación) }\end{array}$ \\
\hline
\end{tabular}

Algunos rankings intentan jerarquizar todas las universidades del mundo, mientras que otros se concentran en un continente (el caso de Asia's Best Universities 2000) o en un solo país. La mayor cobertura es relevante porque sus variables son, en general, más amplias, de modo de lograr indicadores razonablemente comparables. Ocurre que, a medida que la cobertura geográfica disminuye, aumenta la especificidad de las variables incluidas. 
La cobertura disciplinar se relaciona con la orientación académica de la evaluación que lleva adelante el ranking. Uno con enfoque global intenta ordenar las universidades como un todo (sin distinguir entre disciplinas). El disciplinar, por el contrario, distingue entre especialidades, por tanto, realiza una evaluación para cada una de ellas; por último, el unidisciplinar lo hace para una sola de ellas.

Nuevamente, la clasificación es importante porque el tipo y la especificidad de los indicadores requeridos cambia en cada caso: uno global requiere indicadores más generales para establecer las comparaciones que uno disciplinar, que puede utilizar indicadores distintos para cada disciplina.

La cobertura de ámbito se refiere al sector de la oferta educacional en que se centra el análisis, entre los más comunes de una universidad: pregrado (o profesional), posgrado e investigación.

\subsection{Clasificación según directrices de análisis}

A continuación se clasifica a los 12 rankings de esta muestra según las tres directrices anteriormente descritas.

\begin{tabular}{|c|c|c|c|c|c|c|c|c|c|}
\hline & \multicolumn{3}{|c|}{$\begin{array}{l}\text { Cobertura } \\
\text { geográfica }\end{array}$} & \multicolumn{3}{|c|}{$\begin{array}{l}\text { Cobertura } \\
\text { disciplinar }\end{array}$} & \multicolumn{3}{|c|}{$\begin{array}{l}\text { Cobertura de } \\
\text { ámbito }\end{array}$} \\
\hline & 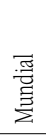 & $\begin{array}{l}\widetilde{\Xi} \\
\widetilde{\Xi} \\
\widetilde{\Xi} \\
\widetilde{x}\end{array}$ & 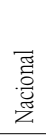 & $\begin{array}{l}\text { 丞 } \\
\text { ज }\end{array}$ & 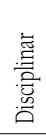 & 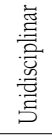 & 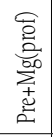 & $\begin{array}{l}\text { o } \\
\text { 행 } \\
0 \\
0\end{array}$ & 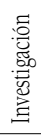 \\
\hline Times Higher World University Ranking & $x$ & & & $\mathrm{X}$ & & & $\mathrm{x}$ & & \\
\hline $\begin{array}{l}\text { Academia Ranking of World Universities - Shanghai Jiao Tong } \\
\text { University }\end{array}$ & $\mathrm{x}$ & & & X & & & & & $x$ \\
\hline Universities Ranking on the web - webometrics.com & $\mathrm{x}$ & & & $x$ & & & & & $\mathrm{x}$ \\
\hline Asia’s Best Universities 2000 - Asia Week & & $\mathrm{x}$ & & $\mathrm{x}$ & & & $\mathrm{x}$ & & \\
\hline The Center Research Universities Ranking - The Center & & & X & $\mathrm{X}$ & & & & X & $\mathrm{x}$ \\
\hline Top Research Universities - The Philosophical Gourmet Report & & & X & & $\mathrm{x}$ & & & & $\mathrm{x}$ \\
\hline $\begin{array}{l}\text { US News and World Report Universities Ranking US News \& } \\
\text { World Report }\end{array}$ & & & $x$ & & $X$ & & $x$ & & \\
\hline The Guardian Universities Ranking - The Guardian & & & $\mathrm{X}$ & & $\mathrm{X}$ & & $\mathrm{x}$ & & \\
\hline
\end{tabular}


24 INDICADORES Y ESTÁNDARES INTERNACIONALES DE CALIDAD UNIVERSITARIA - Rodrigo Fernández, Yerko Martínez, Nicolás Velasco

\begin{tabular}{l|l|l|l|l|l|l|l|l|l}
\hline $\begin{array}{l}\text { Ranking of Graduated Programs in Philosophy - The Philosophical } \\
\text { Gourmet Report }\end{array}$ & & $\mathrm{X}$ & & & & $\mathrm{X}$ & & $\mathrm{X}$ & \\
\hline The Best business schools - University of Texas & & & $\mathrm{X}$ & & & $\mathrm{X}$ & & & \\
\hline MBA Ranking - Financial Review Boss & & & $\mathrm{X}$ & & & $\mathrm{X}$ & $\mathrm{X}$ & & \\
\hline The Sunday Times University Guide - The Sunday Times & & & $\mathrm{X}$ & $\mathrm{X}$ & & & $\mathrm{X}$ & & \\
\hline
\end{tabular}

\section{Conclusiones}

Solo tres de los estudiados intentan evaluar universidades de todo el mundo. Dos de ellos (The Times Higher World University Ranking y The Academia Ranking of World Universities), debido a su enfoque global, usan variables que han sido ampliamente utilizadas en la literatura para medir "calidad". En tanto que el último de ellos (World Universities Ranking on the $W e b^{4}$ ) se centra solamente en el desarrollo de las páginas web de las universidades como plataforma para la difusión y promoción de la investigación.

Considerando los seis con cobertura global (The Times Higher University Ranking, Academia Ranking of World Universities, World Universities Ranking on the web, Asia's Best Universities 2000, The Center Research Universities Ranking y The Sunday Times University Guide), se observa que, en general, los indicadores más utilizados son:

- Razón profesores/estudiantes.

- Calificaciones de entrada, es decir, notas de estudios secundarios y pruebas de selección universitaria.

- Número de citaciones y/o publicaciones (ajustado por el número de profesores de jornada completa equivalente, en el caso de The Times..., o en números brutos en el caso de The Academia... y The Sunday...).

- Evaluación de pares y/o evaluación de excelencia académica.

Sobre los unidisciplinares (Ranking of Graduated Programs in Philosophy ${ }^{5}$, The Best Business Schools ${ }^{6}$ y MBA Ranking), se debe precisar

4 Disponible en http://www.webometrics.info/methodology.htm

5 Disponible en http://www.philosophicalgourmet.com/

6 Disponible en http://www.forbes.com/2003/09/24/bschooland.htm 
que el primero se basa en una encuesta y, por lo tanto, un 100\% de su puntaje se debe al prestigio, mientras que The Best Business Schools se centra un $100 \%$ en el número de publicaciones para crear el ranking de las mejores escuelas de negocios de EE.UU. O sea, sólo en su capacidad de investigación.

Otra precisión debe hacerse con respecto a dos con cobertura de ámbito en investigación: The Center Research Universities Ranking y Top Research Universities ${ }^{7}$, los que, curiosamente, no utilizan indicadores de citaciones o número de publicaciones. El primero realiza una evaluación general de la institución con variables de múltiple orientación ${ }^{8}$, en tanto el segundo se centra en la evaluación de los pares.

Una variable que ha adquirido progresivamente más adherencia dentro de la literatura de rankings es la relativa al prestigio. Para ello se utilizan indicadores tales como "peer review", encuestas a profesionales, encuestas a operadores del mundo laboral, entre otros. Cuatro de las doce clasificaciones analizadas acá incorporan variables de prestigio, y se cree que otras las incorporarían de no ser por el costo asociado con la elaboración de dichas encuestas.

En general, a la variable de prestigio se le asigna una ponderación importante. The Times Higher World University Ranking (versión de 2004) le otorga una ponderación de un 50\%; Top Research Universities Ranking se basa en un $100 \%$ en las encuestas, del mismo

7 Disponible en http://www.philosophicalgourmet.com/topresearch.htm

8 Las variables que incluye son: fondos totales y fondos federales de investigación, activos totales, donaciones anuales, miembros de academias nacionales, profesores premiados, doctores becados, postulaciones a posdoctorados, rango de SAT.

Su metodología considera primero sólo las universidades con fondos estatales para investigación superiores a US $\$ 20$ millones al año; luego ordena estas universidades para cada una de las variables; enseguida, suma el número de veces que una universidad está dentro de las 25 primeras para cada una de las nueve variables anteriores y las ordena según ese criterio. Aquellas que estuvieron entre las 25 mejores en todas las variables ocupan el primer lugar. Las que obtuvieron solo ocho de sus variables dentro de las 25 mejores ocupan el segundo, pero son ordenadas según el número de variables que ocuparon los lugares entre 26 y 50 . Y así sucesivamente. 
modo que el Ranking of Graduated Programs in Philosophy. Por su parte, US News \& World Report Universities Ranking incorpora en casi todas las disciplinas que considera una variable relacionada con la evaluación que hacen los pares o los empleadores de alumnos egresados de una escuela.

A pesar de que la evaluación de los pares pareciera ser una buena medida para la calidad de una institución universitaria, el sesgo que aplica el prestigio puede volverla una variable poco limpia, dado que no tiene que ver necesariamente con la calidad efectiva de la educación impartida actualmente por la institución: crear y cambiar el prestigio de una universidad puede tomar varios años, en cambio, variar la calidad efectiva de la educación, mucho menos. Cuando es verdadero, el prestigio se alcanza por los logros en otros indicadores (calidad de egresados, investigación, relevancia de sus profesores, por ejemplo).

Sin embargo, es una variable a la que debemos atender debido a la importancia que le otorgan las publicaciones como la de The Times Higher Education Supplement. El mismo hecho de aparecer un ranking crea prestigio.

Finalmente, los rankings que se consideran en el presente trabajo son los siguientes:

\begin{tabular}{l|l}
\hline $\begin{array}{l}\text { Times Higher World University } \\
\text { Ranking }\end{array}$ & $\begin{array}{l}\text { Ampliamente reconocido, se le considera como el de mayor } \\
\text { relevancia }\end{array}$ \\
\hline $\begin{array}{l}\text { Academia Ranking of World } \\
\text { Universities }\end{array}$ & Da gran ponderación a variables de investigación. \\
\hline Asia's Best Universities 2000 & $\begin{array}{l}\text { Usa una amplia gama de indicadores dentro de su evaluación } \\
\text { (22 en total). } \\
\text { A pesar de esta diversidad, éstos resultan suficientemente } \\
\text { generales como para poder establecer comparaciones a escala } \\
\text { continental. }\end{array}$ \\
\hline $\begin{array}{l}\text { The Sunday Times University } \\
\text { Guide }\end{array}$ & $\begin{array}{l}\text { A pesar de usar algunos indicadores más idiosincrásicos } \\
\text { (por ejemplo, evaluación de la Agencia de Acreditación } \\
\text { Universitaria de UK), debido a su carácter de ranking nacional, } \\
\text { también utiliza variables más generales. }\end{array}$ \\
\hline
\end{tabular}




\begin{tabular}{l|l}
\hline $\begin{array}{l}\text { US News and World Report } \\
\text { Universities Ranking }\end{array}$ & $\begin{array}{l}\text { Tiene una mirada multidisciplinaria de la educación } \\
\text { universitaria. Utiliza una gama muy amplia y rica de variables } \\
\text { para medir la calidad. Además, incorpora indicadores } \\
\text { específicos para cada disciplina que evalúa. }\end{array}$ \\
\hline $\begin{array}{l}\text { The Guardian Universities } \\
\text { Ranking }\end{array}$ & $\begin{array}{l}\text { Del mismo modo que el de US News and World Report, se } \\
\text { rescata su enfoque disciplinar. }\end{array}$ \\
\hline $\begin{array}{l}\text { The Center Research Universities } \\
\text { Ranking }\end{array}$ & $\begin{array}{l}\text { A pesar de que se centra en un solo ámbito (investigación), } \\
\text { considera todas las disciplinas y podría ser considerado en } \\
\text { términos de obtener indicadores para la investigación. }\end{array}$ \\
\hline
\end{tabular}

En términos generales, podemos clasificar las variables utilizadas en estos rankings de la siguiente manera:

\begin{tabular}{|c|c|c|}
\hline Temas & & Variables \\
\hline \multirow{4}{*}{$\begin{array}{l}\text { Formación } \\
\text { profesional }\end{array}$} & $\begin{array}{l}\text { Selectividad del } \\
\text { estudiantado }\end{array}$ & $\begin{array}{l}\text { Calificaciones de entrada } \\
\text { Tasa de aceptación }\end{array}$ \\
\hline & Recursos & $\begin{array}{l}\text { Profesores/alumnos } \\
\text { Nivel presupuestario } \\
\text { Gasto por estudiante } \\
\text { Recursos de biblioteca }\end{array}$ \\
\hline & Éxito de egresados & $\begin{array}{l}\text { Evaluación de los empleados } \\
\text { Salario de egresados } \\
\text { Tasas de empleo }\end{array}$ \\
\hline & Otras & $\begin{array}{l}\text { Tasa de deserción } \\
\text { Valor agregado o calidad de egresados (1) }\end{array}$ \\
\hline Internacionalización & & $\begin{array}{l}\text { № alumnos internacionales } \\
\mathrm{N}^{0} \text { académicos internacionales } \\
\text { Inclusividad }\end{array}$ \\
\hline $\begin{array}{l}\text { Investigación y } \\
\text { doctorado }\end{array}$ & & $\begin{array}{l}\text { Citaciones } \\
\mathrm{N}^{0} \text { de publicaciones } \\
\mathrm{N}^{0} \text { de doctores egresados } \\
\mathrm{N}^{0} \text { de posdoctorados } \\
\text { Gasto en investigación }\end{array}$ \\
\hline Reconocimiento & & $\begin{array}{l}\text { Evaluación de los pares } \\
\text { Premios ganados por alumnos } \\
\text { Profesores ganadores de premios } \\
\mathrm{N}^{0} \text { de académicos que pertenecen a alguna academia } \\
\text { Excelencia académica }\end{array}$ \\
\hline
\end{tabular}


28 INDICADORES Y ESTÁNDARES INTERNACIONALES DE CALIDAD UNIVERSITARIA - Rodrigo Fernández, Yerko Martínez, Nicolás Velasco

\section{Bibliografía}

Accreditation, Certification, and Quality Assurance Institute. (December, 2003) Guidelines for Self-Evaluation and Assessment of Degree Programmes. Available at http://www.acquin.org

Accrediting Commission for Community and Junior Colleges. Eligibility, Candidacy and Initial Accreditation Manual. Agost, 2004. Available at http://www.accjc.org

Australian Quality Agency Limited (2005) Annual Report 2004. Australian Universities Quality Agency. Available at http://www.auqa.edu.au

Burton R. Clark (2004) Sustaining Change in Universities: Continuities in Case Studies and Concepts. Society for Research into Higher Education \& Open University Press.

Comité National d'évaluation (Enseignement supérieur) (November, 2003) Livre des references. Available at http://www.cne-evaluation.frr

Commission d'evaluation de l'enseignement collegial (June, 2000) The Institutional Evaluation: Guide. Available at http://www.ceec.gouv. ac.ca)

Commission on Colleges Southern Association of Colleges and Schools (2004) Principles of Accreditation: Foundations for Quality Enhancement. Available at http://www.sacscoc.org

European Association for Quality Assurance in Higher Education (2005) Standards and Guidelines for Quality Assurance in the European Higher Education Area. Helsinki. Available at http://www.enqa.net/bologna. Iasso

Middle States Commission on Higher Education (2002) Characteristics of Excellence in Higher Education. Philadelphia: 2002 Available at http:// www.msche.org

New England Association of School and Colleges. Commission on Institutions of Higher Education (2005) Standards for Accreditation. Available at http://www.neasc.org

The Higher Learning Commission. Institutional Accreditation: An Overview. Available at http://www.ncahigherlearningcommission.org

The Quality Assurance Agency for Higher Education. (2004) Code of Practice for Assurance of Academic Quality and Standards in Higher Education. September, 2004. Available at http://www.qaa.ac.uk 
The University of Western Australia (2005) UWA Brief 2005. Available at http://www.uwa.edu.au

WASC (2001) Handbook of Accreditation. Available at http://www.accjc.org

World University Rankings (November, 2004) The Times Higher Education Supplement.

Otras fuentes de Información:

ISI Web of Knowledge

http://www.thomsonisi.com

\section{DARA}

Pontificia Universidad Católica de Chile web page: www.puc.c

Webometrics web page: http://www.webometrics.info/methodology.htm

Philosophical Gourmet web page: http://www.philosophicalgourmet.com

Forbes Review web page: http://www.forbes.com/2003/09/24/bschooland.htm

The Times Higher Education Supplement web page: http://www.thes.co.uk worldrankings,

Academia Ranking of World Universities web page: http://ed.sjtu.edu.cn rank/2005/ARWU2005Main.htm

AsiaWeek web page: http://www.asiaweek.com/asiaweek/features universities2000/index.htm]

The Times web page: http://www.timesonline.co.uk/section/0, $8404,00 . \mathrm{htm}$

US News \& World Report web page: http://www.usnews.com/usnews/edu/grad/ rankings/rankindex_brief.php

The Guardian Education web page: http://education.guardian.co.uk universityguide2004

The Center web page: http://thecenter.ufl.edu/research2003.htm.

Recibido: 30 de agosto de 2006

Aceptado: 4 de septiembre de 2006 